This is an Open Access article distributed under the terms of the Creative Commons Attribution-Noncommercial License, which permits unrestricted use, distribution, and reproduction in any noncommercial medium, provided the original work is properly cited.

\title{
HUNTING CIB GALAXIES WHERE THEY LIVE: BREAKING THE CONFUSION LIMIT WITH BLIND LINE SURVEYS
}

\author{
David Clements ${ }^{1}$, Gwenifer Raymond ${ }^{2},{\text { Kate } \text { Isaak }^{2} \text {, and Chris Pearson }}^{3,4}$ \\ ${ }^{1}$ Physics Department, Imperial College London, Prince Consort Road, London SW7 2AZ, UK \\ ${ }^{2}$ Cardiff University, School of Physics \& Astronomy, Queens Buildings, The Parade, Cardiff, CF24 3AA, UK \\ ${ }^{3}$ Space Science and Technology Department, CCLRC Rutherford Appleton Laboratory, Didcot, Oxfordshire, OX11 0QX, UK \\ ${ }^{4}$ Department of Physics, University of Lethbridge, 4401 University Drive, Lethbridge, Alberta, T1J 1B1, Canada
}

\begin{abstract}
The galaxies responsible for the Cosmic Infrared Background (CIB) are key targets for the SPICA mission. CIB emission peaks at $\sim 150 \mu \mathrm{m}$. Direct observations of the continuum emission of individual galaxies making up the CIB at this wavelength will be hampered by confusion. For the $3.5 \mathrm{~m}$ SPICA primary we expect a confusion limit of $\sim 5 \mathrm{mJy}$ at $150 \mu \mathrm{m}$. Unfortunately much of the CIB flux is produced by sources fainter than this. SPICA, however, is not limited to continuum observations. We have thus examined the possibility of using blank field line surveys using SPICA-SAFARI in spectrometer mode to detect CIB galaxies by their line emission rather than their continuum emission. We find that such observations can not only detect sources with continuum fluxes up to an order of magnitude below the confusion limit, but are also able to measure source spectroscopic redshifts for most objects and offer the potential for far-IR line diagnostics in some. The redshift distributions determined from these observations can be used to directly test galaxy evolution models. We conclude that blank field line surveys are a new and uniquely powerful observing mode for SPICASAFARI, with the potential of revealing much about the galaxies responsible for the CIB.
\end{abstract}

Key words: Galaxies: formation - Galaxies: far-IR - Missions: SPICA

\section{INTRODUCTION}

The discovery of the Cosmic Infrared Background (CIB) (Puget et al., 1996; Fixsen et al., 1998), demonstrated the importance of the far-IR in understanding the formation and evolution of galaxies. The total energy density in the CIB is comparable to that found in the integrated UV/ optical/ near-IR background. Since the CIB is produced by light reprocessed in obscured systems and the $\mathrm{UV} /$ optical/near-IR is produced directly by stellar and accretion processes, their comparable energy densities suggests that roughly $50 \%$ of the energy production in the history of the universe takes place in obscured systems. This is very different from the situation locally, where only $\sim 30 \%$ of the bolometric luminosity of galaxies emerges in the far-IR. Observations with submm bolometer arrays (eg. Smail et al., 1997; Eales et al., 2000) started to probe the individual sources responsible for the longer wavelength end of the CIB spectral energy distribution (SED). These observations resolved $\sim 20 \%$ of the CIB into individual sources but, more importantly, found that the submm bright CIB sources were a radically different population to those responsible for the UV/optical/near-IR (Hughes et al., 1998). To understand this population we thus need to find and examine CIB sources at wavelengths where they luminous.

Other than bolometer arrays, to date much of our knowledge of the CIB population has come from Spitzer. This has come, for example, through studies of broad band spectral energy distributions (SEDs) of submm-detected CIB sources at other wavelengths (Clements et al., 2008; Dye, 2008) and followup observations of such sources with IRS spectroscopy (eg. Coppin et al. in prep). Studies of individual CIB objects have largely been driven by the submm since Spitzer MIPS $160 \mu \mathrm{m}$ observations are too confused to pinpoint more than a small fraction of CIB sources. This was also true of ISO (eg. Puget et al., 1999). An alternative approach to studies of individual sources is the statistical analysis of stacked fluxes, allowing the contribution of populations of sources detected individually at shorter wavelengths, eg. $24 \mu \mathrm{m}$, to the CIB to be assessed (eg. Serjeant et al., 2008). The overall picture that has emerged from all these studies is that the CIB is produced by luminous dust-dominated galaxies lying at $1<\mathrm{z}<3$.

The Herschel Space Observatory, launched in May 2009 (Pilbratt, 2003), is capable of making large area surveys to very faint fluxes at wavelengths from 70 to $500 \mu \mathrm{m}$. It has a $3.5 \mathrm{~m}$ primary mirror, similar in size to that planned for SPICA. However, Herschel will still be confusion limited by the high density of CIB sources for observations around the peak of the CIB SED. Dole et al. 2004 use extrapolations from Spitzer surveys to estimate that the confusion limit of Herschel's PACS instrument is $\sim 5 \mathrm{mJy}$ in its $170 \mu \mathrm{m}$ channel closest to the peak of the CIB SED. Herschel will thus be able to resolve only $\sim 50 \%$ of the CIB into individual sources. Statistical approaches, as applied to Spitzer data, can provide insights into the rest of the population, but these leave considerable uncertainties in the properties of CIB galaxies due to the necessary extrap- 
olation from sources detected at shorter wavelengths (typically $24 \mu \mathrm{m}$ for Spitzer and $70 \mu \mathrm{m}$ for Herschel). Figure 1 shows the ratio of $170 \mu \mathrm{m}$ to 24 or $70 \mu \mathrm{m}$ flux for a range of galaxy template SEDs (see Clements et al., 2007, for details). A full understanding of CIB sources thus needs observations around the peak of the SED at $\sim 150 \mu \mathrm{m}$, just where confusion stops us seeing a large fraction of the population. SPICA, which will have a similarly sized primary to Herschel, will suffer from the same problem, even though it will be sensitive enough to obtain a spectrum for every individual source detected by Herschel. If SPICA is to do more than just followup CIB sources detected by Herschel, then some method is needed to break through the continuum confusion limit. We report here on progress in our examination of the use of far-IR blind line surveys to achieve this goal.

\section{Simulating Blank Field Spectroscopic Surveys}

The huge increase in sensitivity that SPICA has over Herschel, combined with the imaging spectroscopic capabilities of SAFARI, means that we can consider blank field deep spectroscopic surveys. These allow a field to be imaged as a data cube, with wavelength as an additional dimension to the $\mathrm{x}, \mathrm{y}$ position of a source in the field. A narrow band image of the target field can thus be obtained at any given wavelength accessible to the SAFARI instrument. Since far-IR emission lines are expected to be strong and plentiful (eg. Negishi et al., 2001) there is the potential for detecting galaxies by these and not by their continuum emission. The contrast between a narrow band emission line and the broad band continuum confusion noise will be much greater in a narrow band image at the wavelength of the emission line, potentially allowing faint, line emitting galaxies to be detected as individual sources. This approach has the potential to break the traditional continuum confusion limit and permit study of sources which would not otherwise be detected in a continuum survey.

We simulate such observations using the galaxy templates and both the 'burst mode' and 'bright end' evolutionary models discussed in Pearson \& Khan (2009) to which far-IR lines have been added in line with observations (Negishi et al., 2001) together with a simple model of the SAFARI instrument (8"x8" pixels, a 16x16 pixel field of view, fixed spectral resolution of $\Delta \lambda \sim 0.176 \mu \mathrm{m}$ per channel from 30 to $210 \mu \mathrm{m}$, with $0.4 \mathrm{mJy} 1 \sigma$ noise in each spectral channel). Details of the models can be found in Clements et al. (2007) and Raymond et al. (2009). Observations with SPICA-SAFARI were simulated over a 1 sq. deg. region, with subsections of this region analyzed as if they were part of specific SPICA observations. An automated line detection and extraction system allowed sources to be identified, line and continuum fluxes to be extracted and redshifts to be measured from this data (see Raymond et al., 2009, and Raymond et al. this volume for
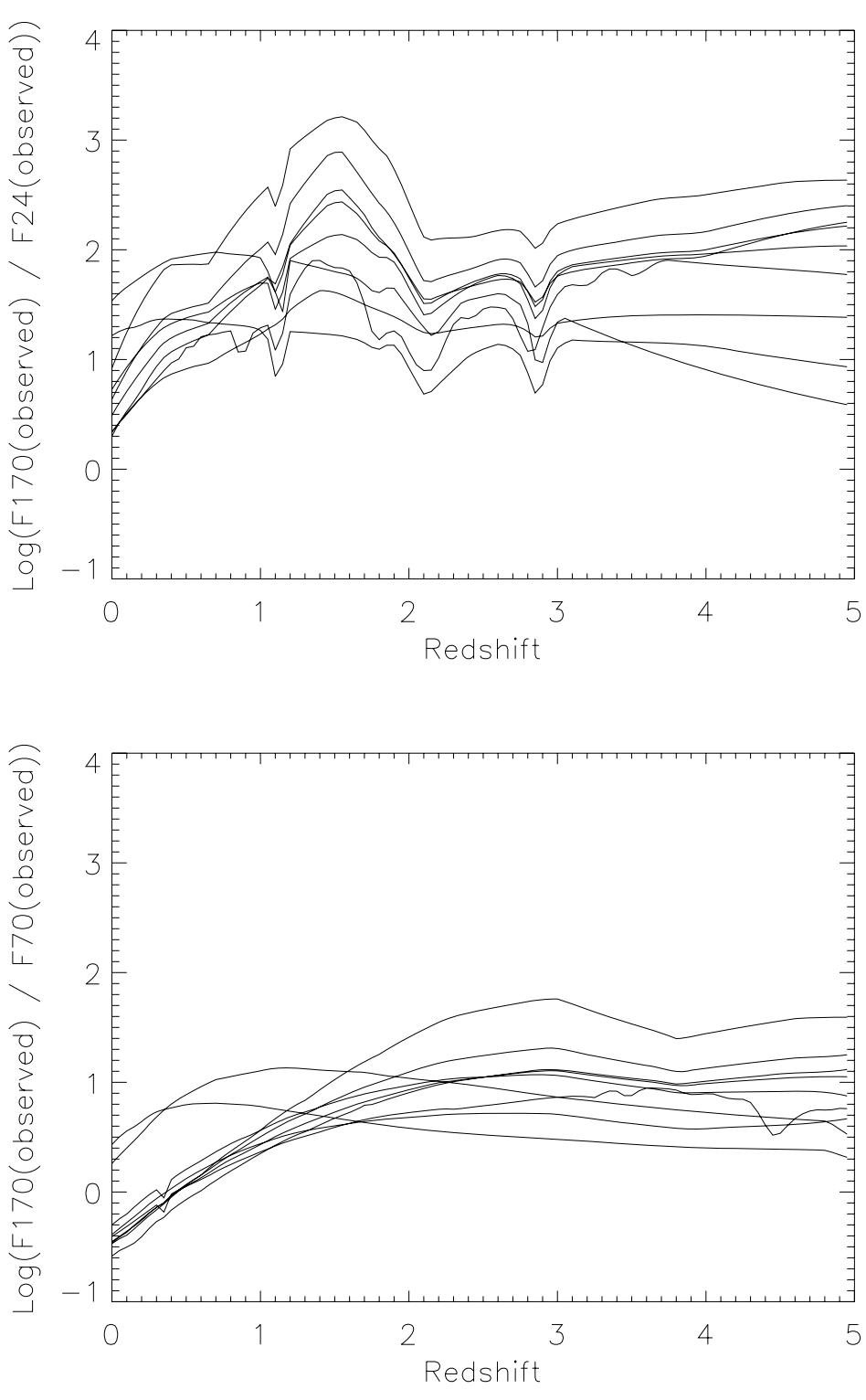

Figure 1. Ratio of SED peak flux to flux detected at another wavelength for a variety of galaxy SEDs.

details). The results of these simulated observations can then be compared to a truth catalog from the input simulations to see how well the technique has performed.

The first, and most important, result of these simulations is the demonstration that we can indeed break the continuum confusion limit with such blank field line surveys. Figure 2 shows the detection of a $0.56 \mathrm{mJy}$ continuum source in line emission compared to the same field seen in continuum emission. No individual source can be identified against the continuum confusion noise in the continuum image, but the faint source is clearly detected in line emission. This source is almost an order of magnitude fainter than the confusion noise in this band. The 

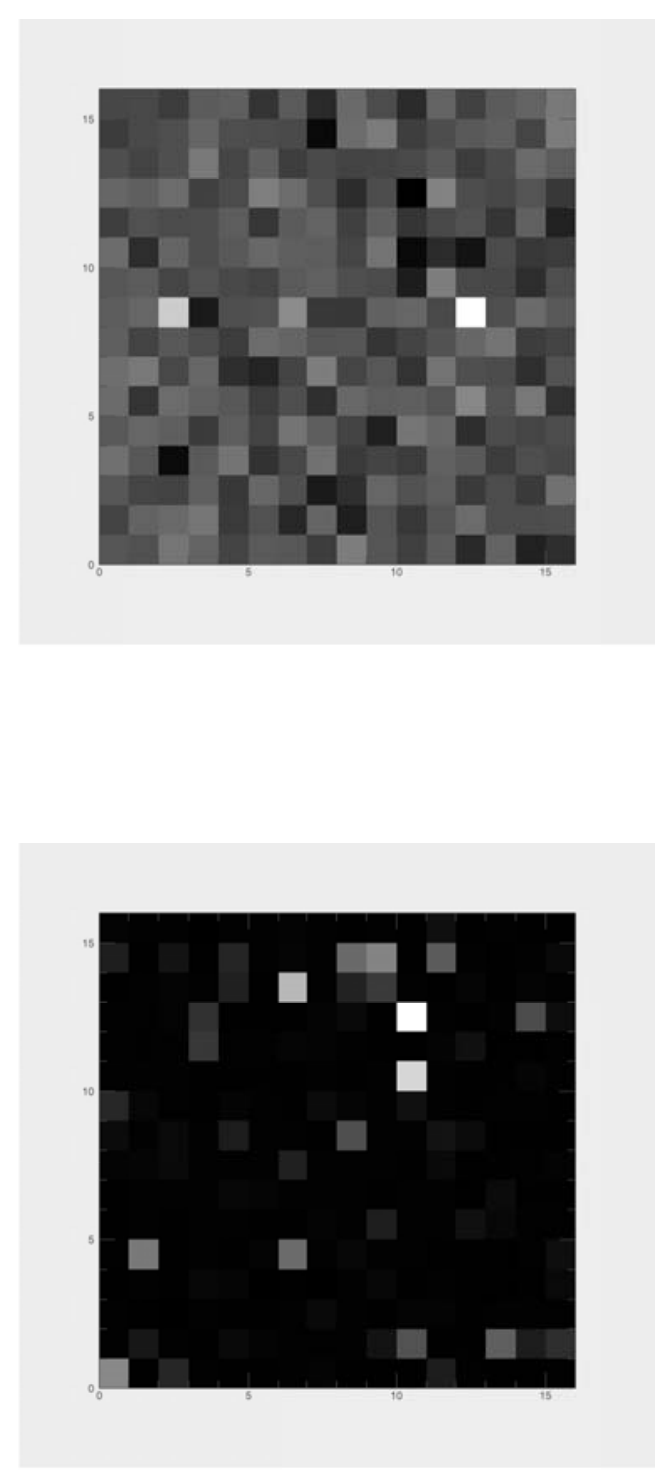

Figure 2. Narrow line image (top) compared to broad band image (bottom). The source clearly detected in the narrow band image has a continuum far-IR flux of $0.56 \mathrm{mJy}$, nearly a factor of 10 below the conventional confusion limit.

basic idea of using narrow-band line imaging to break the confusion limit is clearly demonstrated.

Secondly, we use the catalogs generated by the automated extraction system to examine how this approach performs on large samples of targets and to see if it can be used to distinguish between galaxy evolution models. Figure 3 shows a comparison of recovered vs. input redshifts for our simulated SAFARI observations of the two different Pearson evolution models. In each figure the flux of the recovered source is compared to the confusion noise of the field ie. any source with a $\mathrm{F}_{120} / \mathrm{F}_{c}<1$ is a source fainter than the confusion limit. Clearly we are detecting and measuring redshifts for a great number of sources below the conventional continuum confusion limit. Furthermore, the redshift distributions for these two evolutionary models are different. They are compared using a KS test for simulated results of a survey of 8 SPICA-SAFARI fields ie. $32 \operatorname{arcmin}^{2}$ with 10 hours of integration on each, assuming nominal SPICA-SAFARI sensitivity, ie. 80 hours of observations. We find that we can distinguish between these two evolutionary scenarios at the $99.99 \%$ level of significance with this data set. This is something that would require a survey of a much larger area requiring a greater allocation of observing time if this were to be achieved using conventional number count analysis applied to a continuum survey.

\section{Conclusions}

We have investigated the potential for using blank field far-IR spectroscopic surveys with SPICA-SAFARI to probe the population of galaxies making up the CIB below the continuum confusion limit using simulated observations of model far-IR galaxy populations. We find that sources up to an order of magnitude fainter than the confusion limit can be recovered by this method, and that their redshifts can be measured through the lines in which they are detected. This approach allows us to examine the galaxies responsible for producing the $50 \%$ of CIB flux that will not be individually resolved by continuum measurements at the peak of the CIB SED. We also show, by comparing the results of simulated observations of two different galaxy evolution models, that this technique can very effectively distinguish between different galaxy evolution models with even moderate allocations of time on an instrument such as SAFARI on SPICA. We conclude that blank field line surveys are a new and uniquely powerful observing mode for SPICA-SAFARI.

\section{ACKNOWLEDGEMENTS}

This work has been supported in part by funding from STFC and PPARC.

\section{REFERENCES}

Clements, D.L., Isaak, K.G., Madden, S.C. \& Pearson, C., 2007, A\&A, 465, 125

Clements, D.L. et al. 2008, MNRAS, 387, 247

Dole, et al. 2004, ApJS., 154, 93

Dye, S. et al. 2008, MNRAS, 386, 1107

Eales, S.A. et al. 2000, AJ., 120, 2244

Fixsen, D.J. et al. 1998, ApJ., 508, 123

Hughes, D.H. et al. 1998, Nature, 394, 241

Negishi, T., Onaka, T., Chan, K.-W., Roellig, T. L., 2001, A\&A, 375, 566

Raymond, G., et al. 2009, PASJ, submitted

Pearson, C., \& Khan, S.A., 2009, MNRAS, 399, L11 

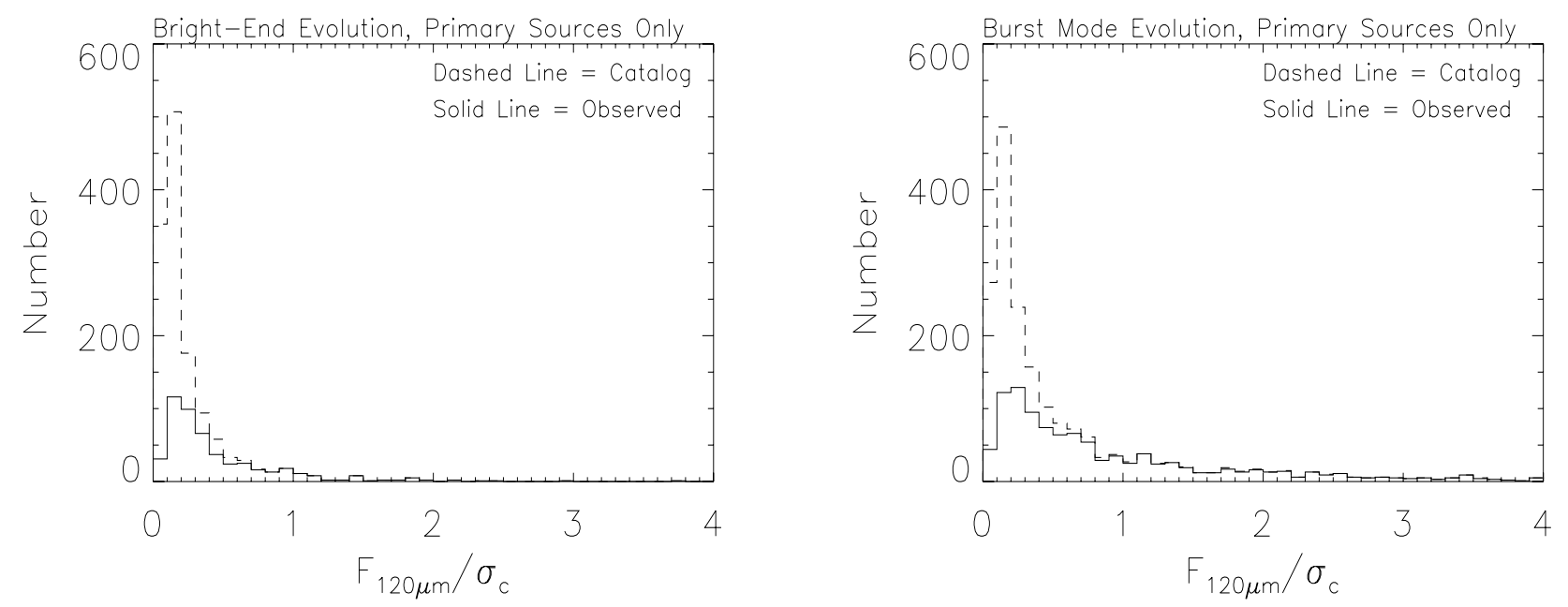

Figure 3. Input and recovered redshift distributions compared to broadband source flux/confusion limit flux. As can be seen many sources and redshifts are recovered below the conventional confusion limit demonstrating the power of the blank field spectroscopic survey technique.

Pilbratt, G. L., 2003 IR Space Telescopes and Instruments. Edited by John C. Mather. Proceedings of the SPIE, 4850, 586

Puget J.-L., et al. 1996, A\&A, 308, L5

Puget J.-L., et al. 1999, A\&A, 345, 29

Serjeant, S., et al. 2008, MNRAS, 386, 1907

Smail, I., Ivison, R., Blain, A.W., 1997, ApJ, 490, L5 